Copyright (C) 2020 University of Bucharest Printed in Romania. All rights reserved

ISSN print: $1224-5984$

ISSN online: $2248-3942$
Rom Biotechnol Lett. 2020; 25(1): 1208-1215

doi: $10.25083 / \mathrm{rbl} / 25.1 / 1208.1215$

\title{
The analysis of the potential for biomass exploitation in the context of bio-economy. Case study: wheat and maize crops
}

\author{
ELENA COFAS ${ }^{1, *}$, ELENA TOMA ${ }^{1}$, ANA URSU $^{2}$, CARINA DOBRE $^{1}$ \\ ${ }^{1}$ University of Agronomic Science and Veterinary Medicine of Bucharest, Faculty of Management, \\ Economic Engineering in Agriculture and Rural Development, Bucharest, Romania \\ ${ }^{2}$ Institute for Agriculture Economy and Rural Development, Bucharest, Romania
}

\begin{abstract}
The future evolution of the energy sector, the challenges faced by industry, the growth premise, and the role that agriculture plays as a source of energy are key elements of the bio-economy. Biomass production in the vegetal agricultural sector is in line with the concept of sustainable development, reduces carbon dioxide emissions through photosynthetic processes and ensures the development of raw materials and renewable energy resources. This article proposed both an economic analysis of biomass potential, represented by the secondary production obtained in wheat and maize crops, for the period 2008-2017, as well as an estimation of the market value of the secondary production correlated with the potential for capitalizing those two crops. The exploitation of the potential of the secondary production is reflected in the production of bioenergy (thermal, electric) and biofuels, respectively, both for use as animal feed and fertilization of agricultural land, as well as non-polluting biofuels.
\end{abstract}

Keywords Bioeconomy, potential, biomass, wheat, maize.

To cite this article: COFAS E, TOMA E, URSU A, DOBRE C. The analysis of the potential for biomass exploitation in the context of bio-economy. Case study: wheat and maize crops. Rom Biotechnol Lett. 2020; 25(1): 1208-1215. DOI: 10.25083/rb1/25.1/1208.1215

*Corresponding author: ELENA COFAS, University of Agronomic Science and Veterinary Medicine of Bucharest, Faculty of Management, Economic Engineering in Agriculture and Rural Development, Bucharest, Romania

E-mail: cofasela@yahoo.com 


\section{Introduction}

Biomass includes any renewable material of organic nature, including terrestrial vegetation (crops for food and feed use, woody vegetation, energy crops, industrial plants), aquatic (algae, herbaceous) and microorganisms (fungi, yeasts, bacteria), as well as organic waste and residues from agriculture, fish farming, forestry, municipal waste and other waste (USDA [13]). Primary biomass is produced by plant photosynthetic activity, representing the assembly of more or less rapid plant raw materials, directly used, or following a conversion process, in human food, feed, various industries, or for the production of energy (EPURE [8]). The main types of biomass conversion processes can be classified into four groups (PANOUTSOU \& al [2]):

$>$ physical (grinding, separation, drying, briquetting, etc.);

> biological / biochemical (fermentation: anaerobic, aerobic, alcoholic);

$>$ thermal (combustion, pyrolysis, gasification, hydrogenation);

$>$ chemical (e.g. biodiesel production from vegetable / animal oils and fats).

Biomass, as a strategic resource, is an important economic factor because it is a continuous, renewable and practical, inexhaustible energy, but also a guarantor of safety and economic sustainability. It is practically accessible to any area and offers both vital products (e.g. food, feed, raw materials for various industries, biofuels and others) as well as socio-economic development, especially in rural areas (COFAS \& TOMA [3]). The need to integrate both renewable resources and the elements that involve the production, transformation and use of biological materials and products has led to the emergence of bioeconomy (we can say it means "biologizing the economy" (EICKHOUT [1]). In the Romanian National Strategy for Research, Development and Innovation 2014-2020, bioeconomy is considered a national area of intelligent specialization. There are many aspects related to the emergence of bio-economy:

- increasing biomass and increasing demand for biomass generation and refinement in industry;

- waste growth has led to innovative initiatives linked, for example, to the integration of livestock production into the values of other industries (TOMA \& al [5]);

- "emerging greenhouse gas mitigation markets boost incentives for biomass stocks (rather than food production) to seize and regenerate the biosphere" (VON BRAUN [7]).

All programmatic documents related to structural and national strategies refer to the agricultural potential (which means, first of all, field crop cultivation), respectively to the secondary production obtained from cereals, as a primary source of biomass. In the budget of any culture, both the value of the main production and the secondary production are highlighted - this can be capitalized both in the field of agricultural production and outside the agricultural production sector. Among different sources of biomass, the crop biomass both as residues or energy plants is one of great importance. Energy plants could be of interest especially when do not compete with food and feed plants, as well as when they put into value the marginal lands or they are using the periods between crop cycles (EUROPABIO [9]). Based on all these considerations, the energy potential of secondary production falls into the energy market in agriculture.

\section{Materials and Methods}

The working methodology of this paper implied the implementation of some activities oriented towards the utilization of bioenergy in agriculture, by means of biotechnology-specific conversion methods, having as a basis the four value chains defined at european level (EUROPEAN COMMISSION [10]):

$\checkmark$ value chain 1 (LC1) - "From lignocellulosic raw materials to advanced biofuels, chemicals made from bioresources and biomaterials";

$\checkmark$ value chain 2 (LC2) - "The new generation of value chain based on forestry";

$\checkmark$ value chain 3 (LC3) - "The new generation of value chain on agricultural bases";

$\checkmark$ value chain 4 (LC4) - "New emerging value chains (organic) waste".

Romanian National strategies mainly refer to LC3, as the potential of value chains associated with agriculture / field crops is obviously underutilized, so that for Romania it is possible to delimit an area of agro-bio-economy, respectively of value chains which is based on plant production. Starting from the agricultural base of the LC3 value chain, this paper proposed both an estimation of the potential of the secondary production (as a source of biomass), represented by the secondary productions obtained during 2008-2017 in the wheat and maize crops, as well a estimate of the market value of secondary production compared to the production potential of the two crops. As inputs, the series of data recorded during 20082017 (INS source) were used for the following variables:

$\rightarrow$ the average annual yields (mean yield, expressed in kilograms per hectare), from which the maximum yield, minimum yield and production value range (as the difference between the maximum yield and the minimum production) were analyzed and the average (as indicator for central trend characterization), respectively the standard deviation (as an indicator for characterization of variability as a measure of the data set dispersion);

$\rightarrow$ the average values of usable agricultural land (average UAA - thousands of hectares);

$\rightarrow$ the average annual purchase prices (RON/kilogram) and the annual values of the consumer price index (CPI); using that index were calculated the average purchase prices, updated at the level of 2017 for the wheat and maize production. Subsequently, these prices were applied to the coefficients related to the secondary production of wheat and maize and the average prices of the secondary productions, updated at the level of 2017, were obtained according to the following formula: 
price secondary production $=$ average purchase price $x$ coefficient secondary production where the secondary production coefficient is 0.15 for wheat and 0.045 for maize (source: ICEADR Bucharest).

Table 1. Input variables

\begin{tabular}{|c|c|c|c|c|c|c|c|c|c|c|c|c|}
\hline Regions & 2008 & 2009 & 2010 & 2011 & 2012 & 2013 & 2014 & 2015 & 2016 & 2017 & Mean & Std. \\
\hline \multicolumn{13}{|c|}{ Wheat - Average yield (kg/ha) } \\
\hline Centre & 3283 & 2789 & 2753 & 3656 & 2647 & 3312 & 3699 & 3736 & 3795 & 4263 & 3314 & 542.9 \\
\hline North-East & 3205 & 2615 & 2696 & 3366 & 2317 & 3173 & 3340 & 3029 & 3609 & 4313 & 3037 & 653.3 \\
\hline North-West & 3445 & 2228 & 2920 & 3620 & 2968 & 3811 & 3843 & 3891 & 3380 & 4255 & 3340 & 616.3 \\
\hline South & 3535 & 2330 & 2621 & 3822 & 2669 & 3772 & 3746 & 4039 & 4110 & 5127 & 3366 & 1010.5 \\
\hline South-East & 3572 & 2142 & 2725 & 3732 & 2353 & 3224 & 3486 & 3702 & 4202 & 5060 & 3238 & 973.3 \\
\hline South-West & 3054 & 2607 & 2478 & 3219 & 2396 & 2831 & 3197 & 3326 & 3315 & 4735 & 2904 & 900.2 \\
\hline West & 3437 & 2806 & 2993 & 4032 & 3878 & 4342 & 4005 & 4451 & 4658 & 5274 & 3874 & 773.2 \\
\hline \multicolumn{13}{|c|}{ Maize - Average yield (kg/ha) } \\
\hline Centre & 3845 & 3826 & 4428 & 4668 & 2542 & 4299 & 5147 & 4025 & 4747 & 5767 & 4204 & 881.6 \\
\hline North-East & 3676 & 3399 & 3881 & 4239 & 1976 & 4541 & 4967 & 3147 & 3569 & 5594 & 3673 & 1161.5 \\
\hline North-West & 4037 & 3429 & 4275 & 4425 & 2730 & 4269 & 4621 & 3156 & 4263 & 5912 & 3991 & 882.5 \\
\hline South & 3026 & 3824 & 4583 & 4963 & 2552 & 5199 & 5294 & 4067 & 4162 & 6643 & 4114 & 1467.0 \\
\hline South-East & 2409 & 2451 & 4337 & 4759 & 1677 & 4261 & 4526 & 3313 & 3939 & 5859 & 3490 & 1423.1 \\
\hline South-West & 2843 & 3801 & 4303 & 4061 & 1458 & 4257 & 4137 & 2958 & 3433 & 6410 & 3504 & 1421.2 \\
\hline West & 3589 & 3498 & 4557 & 4480 & 2890 & 4201 & 4711 & 3703 & 5688 & 5300 & 4114 & 908.1 \\
\hline \multicolumn{13}{|c|}{ Wheat - Average UAA (thou ha) } \\
\hline Centre & 102.2 & 108.8 & 92.7 & 99.1 & 76.3 & 90.0 & 91.3 & 89.5 & 90.7 & 88.6 & 93.3 & 8.1 \\
\hline North-East & 167.4 & 161.9 & 156.2 & 137.3 & 182.9 & 152.7 & 158.4 & 168.0 & 167.8 & 149.3 & 161.0 & 11.6 \\
\hline North-West & 124.5 & 124.6 & 116.2 & 120.1 & 118.2 & 119.5 & 122.9 & 146.6 & 144.7 & 142.9 & 128.6 & 10.9 \\
\hline South & 613.0 & 644.3 & 628.7 & 600.0 & 637.6 & 597.6 & 590.6 & 585.4 & 588.0 & 582.4 & 602.5 & 24.6 \\
\hline South-East & 501.8 & 497.9 & 528.2 & 421.6 & 466.2 & 515.0 & 507.0 & 499.9 & 479.8 & 452.7 & 476.7 & 43.8 \\
\hline South-West & 379.2 & 381.8 & 403.7 & 350.0 & 311.3 & 387.2 & 382.0 & 363.8 & 399.7 & 404.8 & 378.0 & 26.4 \\
\hline West & 207.0 & 215.1 & 219.3 & 203.5 & 186.8 & 222.5 & 242.2 & 234.8 & 248.7 & 214.1 & 220.0 & 17.0 \\
\hline \multicolumn{13}{|c|}{ Maize - Average UAA (thou ha) } \\
\hline Centre & 135.5 & 137.9 & 131.6 & 144.8 & 160.1 & 149.8 & 153.3 & 160.8 & 158.8 & 162.4 & 149.3 & 10.4 \\
\hline North-East & 442.7 & 434.2 & 428.9 & 463.9 & 494.6 & 453.1 & 455.3 & 475.6 & 479.7 & 442.2 & 459.3 & 20.6 \\
\hline North-West & 250.0 & 214.6 & 256.5 & 239.7 & 237.5 & 256.5 & 265.4 & 276.8 & 279.5 & 254.4 & 258.1 & 23.5 \\
\hline South & 478.7 & 440.4 & 391.7 & 479.9 & 544.1 & 484.6 & 462.5 & 499.9 & 470.8 & 454.6 & 472.5 & 36.2 \\
\hline South-East & 455.5 & 429.4 & 335.9 & 515.8 & 500.1 & 481.7 & 469.7 & 471.8 & 475.3 & 434.4 & 457.2 & 45.3 \\
\hline South-West & 382.1 & 382.3 & 276.4 & 386.5 & 414.3 & 346.6 & 349.3 & 360.5 & 362.1 & 324.0 & 358.1 & 34.6 \\
\hline West & 285.5 & 288.7 & 265.1 & 350.5 & 372.2 & 335.5 & 347.5 & 348.6 & 343.6 & 319.6 & 319.9 & 36.5 \\
\hline \multicolumn{13}{|c|}{ Secondary production price* $(\mathrm{RON} / \mathrm{kg})$, constant prices $(2017=100)$} \\
\hline Wheat & 0.111 & 0.071 & 0.083 & 0.118 & 0.123 & 0.118 & 0.112 & 0.114 & 0.097 & 0.098 & $\begin{array}{c}0.106 \\
(0.024 \text { euro })\end{array}$ & $\begin{array}{c}0.016 \\
-\end{array}$ \\
\hline Maize & 0.036 & 0.022 & 0.024 & 0.032 & 0.035 & 0.031 & 0.027 & 0.028 & 0.029 & 0.027 & $\begin{array}{c}0.029 \\
(0.007 \text { euro })\end{array}$ & $\begin{array}{c}0.004 \\
-\end{array}$ \\
\hline
\end{tabular}

Data source: 1998-2018 National Institute of Statistics; $* 1$ euro $=4.5$ RON

\section{Results and Discussion}

Wheat and maize occupy over half of Romanian arable land (around 4.5 mil hectares). In the last decade (2008-2017), at country level, thewheat yield varied from a value of $1541 \mathrm{~kg} / \mathrm{ha}$ to a value of $4888 \mathrm{~kg} / \mathrm{ha}$ ( $\mathrm{std}$. of $859 \mathrm{~kg} / \mathrm{ha}$ ) and the maize yield from a value of $1526 \mathrm{~kg} / \mathrm{ha}$ to a value of $5959 \mathrm{~kg} / \mathrm{ha}$ (std. of $1183 \mathrm{~kg} / \mathrm{ha}$ ). At regional level, the wheat presented a great variability, from a value of $789 \mathrm{~kg} / \mathrm{ha}$ in South-West Region to a value of $5274 \mathrm{~kg} / \mathrm{ha}$ in West Region (Fig. 1). The situation was similar for maize for which the average yield varied from a minimum of $863 \mathrm{~kg} / \mathrm{ha}$ in South-East Region to a maximum of $6643 \mathrm{~kg} / \mathrm{ha}$ in South Region (Fig. 2).

In 2008-2017 period, the regions Centre and NordWest registered the lowest standard deviation of wheat and maize (around $600 \mathrm{~kg} / \mathrm{ha}$ for wheat and $880 \mathrm{~kg} / \mathrm{ha}$ for maize). Region West had the higher average yield of wheat ( $3874 \mathrm{~kg} / \mathrm{ha}$ ) and the Centre Region the higher average yield of maize (4204 kg/ha) (Fig. 3). The regions from south of the country, which concentrate over $50 \%$ (even till $70 \%$ ) of the cultivated area with wheat and maize, are affected more by variability due to pedoclimatic conditions and lack of irrigations (higher deviations). 


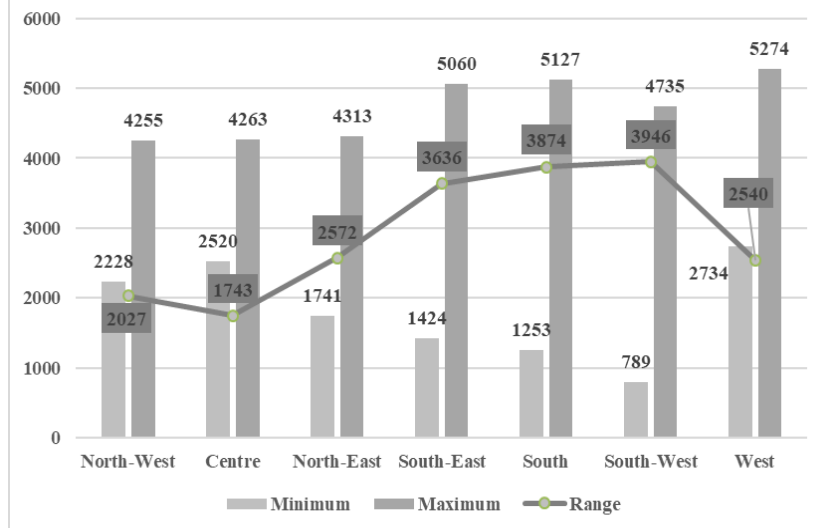

Figure 1. Wheat - Minimum, maximum and range yield (kg/ha), 2008-2017

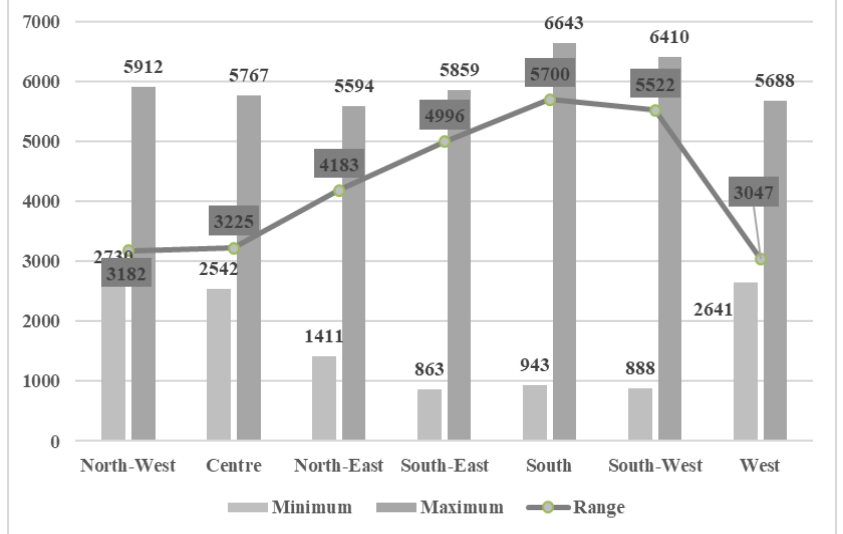

Figure 2. Maize - Minimum, maximum and range yield (kg/ha), 2008-2017

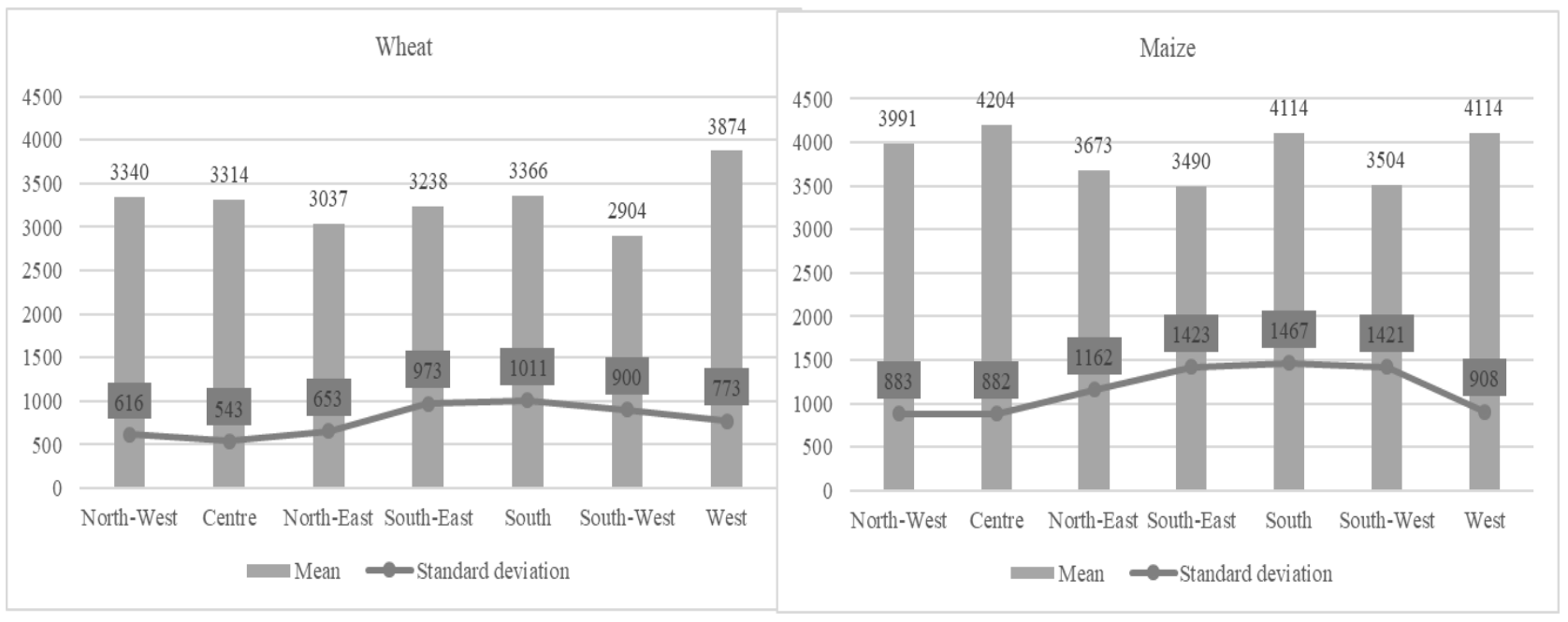

Figure 3. Wheat and maize - Mean and standard deviation - average yield, 2008-2017

In the analysed period, these yield fluctuations, due especially to climatic conditions, lack of irrigation systems and inefficient mix of inputs, represented an impediment in reaching the optimum level of productivity. According with the research studies on around 3000 villages, the maximum potential yield on hectare in our country is for wheat of $5622.3 \mathrm{~kg} / \mathrm{ha}$ and for maize of $8017.9 \mathrm{~kg} / \mathrm{ha}$ and according with Ministry of Agriculture and Rural Development Romania (MADR), official data the crop potential is $3500 \mathrm{~kg} / \mathrm{ha}$ for wheat and $4100 \mathrm{~kg} / \mathrm{ha}$ for maize, in non-irrigated systems, and $6000 \mathrm{~kg} / \mathrm{ha}$ for wheat and maize, in irrigated systems (MADR [11]).

The average values of yield, recorded in 2008-2017 periods at regional level, are between $2600-3200 \mathrm{~kg} / \mathrm{ha}$ for wheat and between 3300-4400 $\mathrm{kg} / \mathrm{ha}$ for maize (Table 2). In these conditions, if we compare the average data from each region with the maximum official level of potential yield, we may observe that we are far away from the maximum level of productivity, even for nonirrigated systems.

Regarding the wheat sector (Table 3), Romania cultivates annually an average UAA of 2.1 mil. ha. In this case, we estimate that the secondary production is in average of 2.4 mil.tons which is under the real potential for this crop, in Romania can be obtain, in optimal conditions (specific for irrigated systems), until $4.3 \mathrm{mil}$. tons from which over $70 \%$ in the southern regions. 
Table 2. Wheat and maize - Share of mean and maximum yields in average and maximum production potential

\begin{tabular}{|c|c|c|c|c|c|c|c|c|c|c|}
\hline \multirow[b]{2}{*}{ Regions } & \multicolumn{5}{|c|}{ Wheat } & \multicolumn{5}{|c|}{ Maize } \\
\hline & $\begin{array}{c}\text { Average } \\
\text { Yield } \\
\text { (kg/ha) }\end{array}$ & $\begin{array}{c}\text { Maximum } \\
\text { potential } \\
\text { yield of } \\
\text { wheat } \\
\text { non- } \\
\text { irrigated } \\
\text { (kg/ha) }\end{array}$ & $\begin{array}{c}\text { Share } \\
(\%)\end{array}$ & $\begin{array}{c}\text { Maximum } \\
\text { potential } \\
\text { yield of } \\
\text { wheat } \\
\text { irrigated } \\
(\mathrm{kg} / \mathrm{ha})\end{array}$ & $\begin{array}{c}\text { Share } \\
(\%)\end{array}$ & $\begin{array}{c}\text { Average } \\
\text { Yield } \\
\text { (kg/ha) }\end{array}$ & $\begin{array}{c}\text { Maximum } \\
\text { potential } \\
\text { yield } \\
\text { of maize } \\
\text { non- } \\
\text { irrigated } \\
\text { (kg/ha) }\end{array}$ & $\begin{array}{c}\text { Share } \\
(\%)\end{array}$ & $\begin{array}{l}\text { Maximu } \\
\mathrm{m} \\
\text { potential } \\
\text { yield of } \\
\text { maize } \\
\text { irrigated } \\
(\mathrm{kg} / \mathrm{ha})\end{array}$ & $\begin{array}{c}\text { Share } \\
(\%)\end{array}$ \\
\hline Centre & 3314 & 3500 & 94.7 & 6000 & 55.2 & 4204 & 4000 & 105.1 & 6000 & 70.1 \\
\hline $\begin{array}{l}\text { North- } \\
\text { East }\end{array}$ & 3037 & 3500 & 86.8 & 6000 & 50.6 & 3673 & 4000 & 91.8 & 6000 & 61.2 \\
\hline $\begin{array}{l}\text { North- } \\
\text { West }\end{array}$ & 3340 & 3500 & 95.4 & 6000 & 55.7 & 3991 & 4000 & 99.8 & 6000 & 66.5 \\
\hline South & 3366 & 3500 & 96.2 & 6000 & 56.1 & 4114 & 4000 & 102.9 & 6000 & 68.6 \\
\hline $\begin{array}{c}\text { South- } \\
\text { East }\end{array}$ & 3238 & 3500 & 92.5 & 6000 & 54.0 & 3490 & 4000 & 87.3 & 6000 & 58.2 \\
\hline $\begin{array}{l}\text { South- } \\
\text { West }\end{array}$ & 2904 & 3500 & 83.0 & 6000 & 48.4 & 3504 & 4000 & 87.6 & 6000 & 58.4 \\
\hline West & 3874 & 3500 & 110.7 & 6000 & 64.6 & 4114 & 4000 & 102.9 & 6000 & 68.6 \\
\hline
\end{tabular}

Table 3. Wheat - Estimation of average secondary production for 2008-2017 compared with the potential

\begin{tabular}{|c|c|c|c|c|c|c|c|c|c|c|}
\hline Regions & $\begin{array}{c}\text { Average } \\
\text { Yield } \\
\text { (kg/ha) }\end{array}$ & $\begin{array}{c}\text { Secondary } \\
\text { production } \\
(\mathrm{kg} / \mathrm{ha})^{*}\end{array}$ & $\begin{array}{c}\text { Maximum } \\
\text { potential } \\
\text { yield of } \\
\text { wheat } \\
\text { Non- } \\
\text { irrigated } \\
(\mathrm{kg} / \mathrm{ha})\end{array}$ & $\begin{array}{c}\text { Secondary } \\
\text { production } \\
(\mathrm{kg} / \mathrm{ha})\end{array}$ & $\begin{array}{c}\text { Maximum } \\
\text { potential } \\
\text { yield of } \\
\text { wheat } \\
\text { Irrigated } \\
(\mathrm{kg} / \mathrm{ha})\end{array}$ & $\begin{array}{c}\text { Secondary } \\
\text { production } \\
(\mathrm{kg} / \mathrm{ha})\end{array}$ & $\begin{array}{c}\text { Average } \\
\text { UAA } \\
\text { (ha) }\end{array}$ & $\begin{array}{c}\text { Total } \\
\text { average } \\
\text { secondary } \\
\text { production } \\
\text { (tones) }\end{array}$ & $\begin{array}{c}\text { Total } \\
\text { secondary } \\
\text { production } \\
\text { potential } \\
\text { Non- } \\
\text { irrigated } \\
\text { (tones) }\end{array}$ & $\begin{array}{c}\text { Total } \\
\text { secondary } \\
\text { production } \\
\text { potential } \\
\text { Irrigated } \\
\text { (tones) }\end{array}$ \\
\hline Centre & 3314 & 1160 & 3500 & 1225 & 6000 & 2100 & 128647 & 149218 & 157593 & 270159 \\
\hline $\begin{array}{c}\text { North- } \\
\text { East }\end{array}$ & 3037 & 1063 & 3500 & 1225 & 6000 & 2100 & 93334 & 99209 & 114334 & 196001 \\
\hline $\begin{array}{l}\text { North- } \\
\text { West }\end{array}$ & 3340 & 1169 & 3500 & 1225 & 6000 & 2100 & 161049 & 188266 & 197285 & 338203 \\
\hline South & 3366 & 1178 & 3500 & 1225 & 6000 & 2100 & 476656 & 561548 & 583904 & 1000978 \\
\hline $\begin{array}{c}\text { South- } \\
\text { East }\end{array}$ & 3238 & 1133 & 3500 & 1225 & 6000 & 2100 & 602542 & 682861 & 738114 & 1265338 \\
\hline $\begin{array}{l}\text { South- } \\
\text { West }\end{array}$ & 2904 & 1016 & 3500 & 1225 & 6000 & 2100 & 377971 & 384170 & 463014 & 793739 \\
\hline West & 3874 & 1356 & 3500 & 1225 & 6000 & 2100 & 220040 & 298352 & 269549 & 462084 \\
\hline TOTAL & & & & & & & 2060239 & 2363625 & 2523793 & 4326502 \\
\hline
\end{tabular}

*Coefficient 0.35, ICEADR Bucharest source

Regarding the maize sector (Table 4), Romania in optimal conditions (specific for irrigated systems), we cultivates annually an average UAA of 2.5 mil. ha. can obtain almost 6.7 mil. tones of secondary production, The secondary production is estimated at an average level concentrated especially in regions South-East, North-West of 4.3 mil. tones, also under the real potential for this crop, and South. 
Table 4. Maize - Estimation of average secondary production for 2008-2017 compared with the potential

\begin{tabular}{|c|c|c|c|c|c|c|c|c|c|c|}
\hline Regions & $\begin{array}{c}\text { Average } \\
\text { Yield } \\
\text { (kg/ha) }\end{array}$ & $\begin{array}{c}\text { Secondary } \\
\text { production } \\
(\mathrm{kg} / \mathrm{ha})^{*}\end{array}$ & $\begin{array}{c}\text { Maximum } \\
\text { potential } \\
\text { yield of } \\
\text { wheat } \\
\text { Non- } \\
\text { irrigated } \\
(\mathrm{kg} / \mathrm{ha})\end{array}$ & $\begin{array}{c}\text { Secondary } \\
\text { production } \\
(\mathrm{kg} / \mathrm{ha})\end{array}$ & $\begin{array}{c}\text { Maximum } \\
\text { potential } \\
\text { yield of } \\
\text { wheat } \\
\text { Irrigated } \\
(\mathrm{kg} / \mathrm{ha})\end{array}$ & $\begin{array}{c}\text { Secondary } \\
\text { production } \\
(\mathrm{kg} / \mathrm{ha})\end{array}$ & $\begin{array}{c}\text { Average } \\
\text { UAA } \\
\text { (ha) }\end{array}$ & $\begin{array}{c}\text { Total } \\
\text { average } \\
\text { secondary } \\
\text { production } \\
\text { (tonnes) }\end{array}$ & $\begin{array}{c}\text { Total } \\
\text { secondary } \\
\text { production } \\
\text { potential } \\
\text { Non- } \\
\text { irrigated } \\
\text { (tonnes) }\end{array}$ & $\begin{array}{c}\text { Total } \\
\text { secondary } \\
\text { production } \\
\text { potential } \\
\text { Irrigated } \\
\text { (tonnes) }\end{array}$ \\
\hline Centre & 4204 & 1892 & 4000 & 1800 & 6000 & 2700 & 258072 & 488221 & 464530 & 696794 \\
\hline $\begin{array}{c}\text { North- } \\
\text { East }\end{array}$ & 3673 & 1653 & 4000 & 1800 & 6000 & 2700 & 149322 & 246807 & 268780 & 403169 \\
\hline $\begin{array}{l}\text { North- } \\
\text { West }\end{array}$ & 3991 & 1796 & 4000 & 1800 & 6000 & 2700 & 459302 & 824883 & 826744 & 1240115 \\
\hline South & 4114 & 1851 & 4000 & 1800 & 6000 & 2700 & 457235 & 846479 & 823023 & 1234535 \\
\hline $\begin{array}{c}\text { South- } \\
\text { East }\end{array}$ & 3490 & 1571 & 4000 & 1800 & 6000 & 2700 & 472509 & 742075 & 850516 & 1275774 \\
\hline $\begin{array}{c}\text { South- } \\
\text { West }\end{array}$ & 3504 & 1577 & 4000 & 1800 & 6000 & 2700 & 358107 & 564663 & 644593 & 966889 \\
\hline West & 4114 & 1851 & 4000 & 1800 & 6000 & 2700 & 319857 & 592151 & 575743 & 863614 \\
\hline TOTAL & & & & & & & 2474404 & 4305280 & 4453927 & 6680891 \\
\hline
\end{tabular}

*Coefficient 0.45, ICEADR Bucharest source

Starting from previous data, for wheat (Table 5) we calculated that the average annual value of secondary products market in of 56.73 mil euro. By not improving the yields, at a level of non-irrigated systems, Romanian wheat sector loosed annually around $4 \mathrm{mil}$ euro and by not reaching the full potential (specific for irrigated systems) loosed around 47 mil euro.
For maize (Table 6) we estimated that the average annual value of secondary products market in of 30.14 mil euro. In our opinion, by improving the yields at least at the level of non-irrigated systems, the maize sector can gain annually around 1 mil euro and by reaching the maximum production on hectare (specific for irrigated systems) it can gain around 17 mil euro.

Table 5. Wheat - Estimation of secondary production market value compared with the potential

\begin{tabular}{|c|c|c|c|c|c|c|c|c|}
\hline Regions & $\begin{array}{c}\text { Total } \\
\text { average } \\
\text { secondary } \\
\text { production } \\
\text { (tons) }\end{array}$ & $\begin{array}{l}\text { Total } \\
\text { secondary } \\
\text { production } \\
\text { potential } \\
\text { Non- } \\
\text { irrigated } \\
\text { (tons) }\end{array}$ & $\begin{array}{l}\text { Total } \\
\text { secondary } \\
\text { production } \\
\text { potential } \\
\text { Irrigated } \\
\text { (tons) }\end{array}$ & $\begin{array}{c}\text { Total } \\
\text { estimated } \\
\text { value of } \\
\text { secondary } \\
\text { production } \\
\text { (mil euros)* }\end{array}$ & $\begin{array}{c}\text { Total } \\
\text { estimated } \\
\text { value of } \\
\text { potential } \\
\text { secondary } \\
\text { production } \\
\text { Non- } \\
\text { irrigated } \\
\text { (mil euros) }\end{array}$ & $\begin{array}{c}\text { Total } \\
\text { estimated } \\
\text { value of } \\
\text { potential } \\
\text { secondary } \\
\text { production } \\
\text { Irrigated } \\
\text { (mil euros) }\end{array}$ & $\begin{array}{c}\text { Gap } \\
\text { from } \\
\text { potential } \\
\text { Non- } \\
\text { irrigated } \\
\text { (mil } \\
\text { euros) }\end{array}$ & $\begin{array}{c}\text { Gap } \\
\text { from } \\
\text { potential } \\
\text { Irrigated } \\
\text { (mil euro }\end{array}$ \\
\hline Centre & 149218 & 157593 & 270159 & 3.58 & 3.78 & 6.48 & 0.20 & 2.90 \\
\hline $\begin{array}{c}\text { North- } \\
\text { East }\end{array}$ & 99209 & 114334 & 196001 & 2.38 & 2.74 & 4.70 & 0.36 & 2.32 \\
\hline $\begin{array}{l}\text { North- } \\
\text { West }\end{array}$ & 188266 & 197285 & 338203 & 4.52 & 4.73 & 8.12 & 0.22 & 3.60 \\
\hline South & 561548 & 583904 & 1000978 & 13.48 & 14.01 & 24.02 & 0.54 & 10.55 \\
\hline $\begin{array}{c}\text { South- } \\
\text { East }\end{array}$ & 682861 & 738114 & 1265338 & 16.39 & 17.71 & 30.37 & 1.33 & 13.98 \\
\hline $\begin{array}{l}\text { South- } \\
\text { West }\end{array}$ & 384170 & 463014 & 793739 & 9.22 & 11.11 & 19.05 & 1.89 & 9.83 \\
\hline West & 298352 & 269549 & 462084 & 7.16 & 6.47 & 11.09 & -0.69 & 3.93 \\
\hline TOTAL & 2363625 & 2523793 & 4326502 & 56.73 & 60.57 & 103.84 & 3.84 & 47.11 \\
\hline
\end{tabular}

*Average price $0.106 \mathrm{RON} / \mathrm{kg}(0.024$ euro $/ \mathrm{kg}), 1$ euro $=4.5 \mathrm{RON}$ 
Table 6. Maize - Estimation of secondary production market value compared with the potential

\begin{tabular}{|c|c|c|c|c|c|c|c|c|}
\hline Regions & $\begin{array}{c}\text { Total } \\
\text { average } \\
\text { secondary } \\
\text { production } \\
\text { (tons) }\end{array}$ & $\begin{array}{c}\text { Total } \\
\text { secondary } \\
\text { production } \\
\text { potential } \\
\text { Non- } \\
\text { irrigated } \\
\text { (tons) }\end{array}$ & $\begin{array}{c}\text { Total } \\
\text { secondary } \\
\text { production } \\
\text { potential } \\
\text { Irrigated } \\
\text { (tons) }\end{array}$ & $\begin{array}{c}\text { Total } \\
\text { estimated } \\
\text { value of } \\
\text { secondary } \\
\text { production } \\
\quad \text { (mil } \\
\text { euros)* }\end{array}$ & $\begin{array}{c}\text { Total } \\
\text { estimated } \\
\text { value of } \\
\text { potential } \\
\text { secondary } \\
\text { production } \\
\text { Non- } \\
\text { irrigated } \\
\text { (mil euros) }\end{array}$ & $\begin{array}{c}\text { Total } \\
\text { estimated } \\
\text { value of } \\
\text { potential } \\
\text { secondary } \\
\text { production } \\
\text { Irrigated } \\
\text { (mil euros) }\end{array}$ & $\begin{array}{c}\text { Gap } \\
\text { from } \\
\text { potential } \\
\text { Non- } \\
\text { irrigated } \\
\text { (mil } \\
\text { euros) }\end{array}$ & $\begin{array}{l}\text { Gap from } \\
\text { potential } \\
\text { Non- } \\
\text { irrigated } \\
\text { (mil euros) }\end{array}$ \\
\hline Centre & 488221 & 464530 & 696794 & 3.42 & 3.25 & 4.88 & -0.17 & 1.46 \\
\hline $\begin{array}{c}\text { North- } \\
\text { East }\end{array}$ & 246807 & 268780 & 403169 & 1.73 & 1.88 & 2.82 & 0.15 & 1.09 \\
\hline $\begin{array}{l}\text { North- } \\
\text { West }\end{array}$ & 824883 & 826744 & 1240115 & 5.77 & 5.79 & 8.68 & 0.01 & 2.91 \\
\hline South & 846479 & 823023 & 1234535 & 5.93 & 5.76 & 8.64 & -0.16 & 2.72 \\
\hline $\begin{array}{c}\text { South- } \\
\text { East }\end{array}$ & 742075 & 850516 & 1275774 & 5.19 & 5.95 & 8.93 & 0.76 & 3.74 \\
\hline $\begin{array}{c}\text { South- } \\
\text { West }\end{array}$ & 564663 & 644593 & 966889 & 3.95 & 4.51 & 6.77 & 0.56 & 2.82 \\
\hline West & 592151 & 575743 & 863614 & 4.15 & 4.03 & 6.05 & -0.11 & 1.90 \\
\hline TOTAL & 4305280 & 4453927 & 6680891 & 30.14 & 31.18 & 46.77 & 1.04 & 16.63 \\
\hline
\end{tabular}

*Average price $0.029 \mathrm{RON} / \mathrm{kg}(0.007$ euro $/ \mathrm{kg}), 1$ euro $=4.5 \mathrm{RON}$

For 2008-2017 periods, the market of secondary products (wheat and maize) is estimated at a value of 868.6 mil euro. Thus the estimated value of potential inputs for bio-based industry reached 567.3 mil euro for wheat and 301.4 mil euro for maize (Table 7).
However, we observe that, if the farms can obtain maximum yields, this sector can reach a value of $1506.0 \mathrm{mil}$ euro, $69 \%$ from wheat sector and $31 \%$ from maize sector. This means that, in the 2008-2017 periods, bio-based industry loosed raw materials of almost 640 mil euro.

Table 7. Estimation of 10 years secondary production market value compared with the potential

\begin{tabular}{|c|c|c|c|c|c|c|c|}
\hline Regions & $\begin{array}{c}\text { Wheat } \\
\text { The estimated } \\
\text { value of bio- } \\
\text { based industry } \\
2008-2017 \\
\text { (mil euros) }\end{array}$ & $\begin{array}{c}\text { Maize } \\
\text { The estimated } \\
\text { value of bio- } \\
\text { based industry } \\
2008-2017 \\
\text { (mil euros) }\end{array}$ & $\begin{array}{c}\text { Total } \\
\text { secondary } \\
\text { production } \\
2008-2017 \\
\text { (mil euros) }\end{array}$ & $\begin{array}{c}\text { Wheat } \\
\text { Market } \\
\text { potential } \\
\text { for } 10 \\
\text { years } \\
\text { (mil } \\
\text { euros) }\end{array}$ & $\begin{array}{c}\text { Maize } \\
\text { Market } \\
\text { potential } \\
\text { for 10 } \\
\text { years } \\
\text { (mil } \\
\text { euros) }\end{array}$ & $\begin{array}{c}\text { Total market } \\
\text { potential for } \\
10 \text { years } \\
\text { (mil euros) }\end{array}$ & $\begin{array}{l}\text { Loss by not } \\
\text { reaching the } \\
\text { potential } \\
\text { (mil euros) }\end{array}$ \\
\hline Centre & 35.81 & 34.18 & 69.99 & 64.84 & 48.78 & 113.61 & 43.62 \\
\hline $\begin{array}{c}\text { North- } \\
\text { East }\end{array}$ & 23.81 & 17.28 & 41.09 & 47.04 & 28.22 & 75.26 & 34.17 \\
\hline $\begin{array}{l}\text { North- } \\
\text { West }\end{array}$ & 45.18 & 57.74 & 102.92 & 81.17 & 86.81 & 167.98 & 65.06 \\
\hline South & 134.77 & 59.25 & 194.02 & 240.23 & 86.42 & 326.65 & 132.63 \\
\hline $\begin{array}{c}\text { South- } \\
\text { East }\end{array}$ & 163.89 & 51.95 & 215.84 & 303.68 & 89.30 & 392.99 & 177.15 \\
\hline $\begin{array}{c}\text { South- } \\
\text { West }\end{array}$ & 92.20 & 39.53 & 131.73 & 190.50 & 67.68 & 258.18 & 126.45 \\
\hline West & 71.60 & 41.45 & 113.05 & 110.90 & 60.45 & 171.35 & 58.30 \\
\hline TOTAL & 567.27 & 301.37 & 868.64 & 1038.36 & 467.66 & 1506.02 & 637.38 \\
\hline
\end{tabular}


From the presented analysis we can see that the biomass, expressed quantitatively by the value of the secondary production, is a promising renewable energy source for Romania, both in terms of its potential and the possibilities of its use. Certainly it can be said that the market potential of biomass is very high, both for wheat and maize. In our opinion, by improving yields, at least at the level of non-irrigated systems, the wheat sector can earn about EUR 4 million a year, and maize can earn about EUR 1 million annually. Extrapolating, by reaching the maximum yield per hectare ((specific to irrigated systems), they can get up to 47 million euros for secondary wheat production and 17 million for maize.

Although secondary production is reduced compared to the main production, it does not reflect the true value of the market, even if only a part of the secondary production is used in the development of these biomass-based raw materials industries. From this analysis it can be deduced that the prospect of superior utilization of biomass by suitable methods may lead to a vision of the use of bio-mass in the context of the national bio-economy.

Research and development policies have identified more needs related to bio-economy, which should materialize in new technologies, biological products, biomass types, production techniques etc. (COFAS \& al [4]). The requirements of the current stage of development for unconventional activities in the rural area impose specific energy market requirements resulting from the capitalization of secondary production. Regarding this problems it can be mentioned some of the advantages resulting from the use of biomass, namely (TOMA [6]):

$>$ obtaining and easy renewal of these products through different conversion methods that can be used to produce energy (thermal, electrical);

$>$ use as animal feed and fertilization of agricultural land;

$>$ use as a fuel that does not raise environmental pollution problems.

Based on all these considerations, the energy potential of the by-products falls into the energy market in agriculture. Limiting valorisation within a particular production system no longer meets current requirements, so that, for this reason, the energy value of secondary agricultural production must be integrated into a global energy market (OCED[12]).

\section{Conclusions}

The economic analysis carried out in this paper highlights the huge potential of secondary production, if it is fully exploited. However, we must bear in mind that the use of this secondary production exclusively as a renewable energy resource would affect the biological balance of the soil. Maintaining and increasing soil fertility are primarily conditioned by the quantity and quality of organic matter in the soil. In the easiest and safest way, sources of organic matter for the soil come from crop residues, ie from secondary agricultural products. Thus, many farmers are not interested in the economical quantification of biomass from grain cultivation and export of secondary production, available as renewable energy resources, because it is more convenient for them to leave these secondary products as organic matter for soil.

In conclusion, the problems of capitalizing on the secondary production in agriculture follow at the present stage tendencies related to a double aspect: first of all one can refer to the existence and accentuation of the energy crisis of the fossil fuels, and secondly to the pollution and protection of the systems ecological. All these aspects mean for the "biomass-energy-ecology" ensemble a new form for the market of energy from secondary agricultural production, considering here also the qualitative aspect regarding the biomass content and the current energy conversion possibilities, which in the end will lead to finding the most appropriate and accessible forms of rational use and valorisation of biomass in the context of building and developing the bio-economy.

\section{References}

1. B. EICKHOUT, A strategy for a bio-based economy, Green New Deal Series, volume 9 (2012).

2. C. PANOUTSOU, J. ELEFTHERIADIS, A. NIKOLAOU "Biomass supply in EU27 from 2010 to 2030", Energy Policy 37 (2009).

3. E.COFAS, E.TOMA, Assessing Gaps in Romanian Agriculture Based on Production Potential of the Main Crops, Proceedings of The 23rd IBIMA Conference, Vision 2020 - Sustainable Growth, Economic Development and Global Competitiveness, Valencia (2014).

4. E. COFAS, M.C. VLAD, R. BEREVOIANU, Organic farming in the context of the bioeconomy, Munich Personal RePEc Archive Paper No. 53499 (2014).

5. E. TOMA, S. CRISTEA, C. DOBRE, I. DONA, From bio-based products to bio-based industries development in an emerging BioEconomy, Romanian Biotechnological Letters, vol. 23 (1): 13350-56 (2018).

6. E. TOMA, I. M. VLAD, Land concentration changes in Romania during 2002-2016 period, AgroLife Scientific Journal - Volume 7, Number 1 (2018).

7. J. VON BRAUN, The role of Science and research for development policy and the millennium development goals, Humboldt Foundation, Berlin (2008).

8. L.I. EPURE, Studiul resurselor de biomasă pe teritoriul României, sub aspectul potențialului agricol și energetic. Teză de doctorat. USAMV București (2006).

9. EUROPABIO (European Association for Bioindustries), Building a Bio-based Economy for Europe in 2020; European Association for Bioindustries: Brussels, Belgium (2011).

10. EUROPEAN COMMISSION, Communication. Innovating for Sustainable Growth: A Bioeconomy for Europe (2012).

11. MADR, Anexa 10 - Zone cu potențial agricol. Anexa II A - Producțiile medii orientative în funcție de potențial ale principalelor culturi (2015).

12. OCED, The Bioeconomy to 2030: designing a policy agenda - International Futures Programme (2009).

13. USDA, Biobased Economy Indicators. A report to the US Congress (2011). 